\title{
ON A PROBLEM IN THE DYNAMIC THEORY OF CRACKS
}

\author{
BY
}

\author{
R. J. TAIT AND T. BRYANT MOODIE \\ University of Alberta, Edmonton
}

\begin{abstract}
We show that it is possible, for a certain case of a traveling crack problem, to obtain an explicit solution, in the entire region of interest, in terms of elementary functions. This affords a simple way of constructing level stress curves in the entire region, in contrast with the general case when simple expressions are obtainable at best along a particular axis.

1. Introduction. In a recent paper [1] we presented a general method of constructing closed-form solutions for a class of elastodynamic problems. In particular this class contained special cases considered by Singh, Moodie, and Haddow [2], who used dual integral equation techniques. In general the methods of [2] have wide applicability, but tend to give simple explicit expressions for the unknown quantities only along a particular axis. In [2] the authors concentrate on the values of the stresses and displacements along the $x$-axis and compute the stress intensity factor at the crack tip. Since, in a case which appears to be of some practical importance, the method of [1] gives explicit expressions in terms of elementary functions throughout the region of interest, we present this particular result, together with the level stress curves.
\end{abstract}

2. Equations and solution. Consider a slab of elastic material with cross section occupying the strip $-\infty<X<\infty,-h<Y<h$ of the $X-Y$ plane, where $O X Y Z$ is a fixed rectangular coordinate system. We assume a crack of width $2 a$ is propagating in the midplane of the strip with velocity $v$ in the positive $X$ direction, in the presence of anti-plane shear. For reference to such problems see Sih and Chen [3] or Sneddon and Lowengrub [4].

Assuming that there is a single non-vanishing component of displacement in the $Z$ direction, we have

$$
U=V=0, \quad W=W(X, Y, t),
$$

where $U, V, W$ are the displacement components in the $X, Y$, and $Z$ directions respectively. Then

$$
\begin{gathered}
\sigma_{X}=\sigma_{Y}=\sigma_{Z}=\sigma_{X Y}=0, \\
\sigma_{X Z}=\mu \frac{\partial W}{\partial X}, \quad \sigma_{Y Z}=\mu \frac{\partial W}{\partial Y},
\end{gathered}
$$

where $\mu$ is the shear modulus and we employ standard notation for stress components.

\footnotetext{
* Received July 1, 1980.
} 
The equations of motion then reduce to

$$
\frac{\partial^{2} W}{\partial X^{2}}+\frac{\partial^{2} W}{\partial Y^{2}}=\frac{1}{\tilde{b}^{2}} \frac{\partial^{2} W}{\partial t^{2}}
$$

where $\tilde{b}=(\mu / \rho)^{1 / 2}$ is the shear wave velocity and $\rho$ the constant density. If it is further assumed that all quantities are functions only of $X-v t$, and $Y$, set

$$
x=X-v t, \quad y=s Y, \quad t=t .
$$

Then (2.4) becomes

$$
\left(\partial^{2} W / \partial x^{2}\right)+\left(\partial^{2} W / \partial y^{2}\right)=0
$$

provided we define $s^{2}=1-v^{2} / \tilde{b}^{2}, v<\tilde{b}$. For future reference we define $\sigma_{y z}=\mu(\partial W / \partial y)$, $\sigma_{x z}=\mu(\partial W / \partial x)$.

The problem now reduces to finding a solution of $(2.6)$ in $(-\infty, \infty) \times(-h s, h s)$ such that

$$
W(x, 0)=0 \quad|x|>a, \quad \sigma_{y z}(x, 0)=0, \quad|x|<a,
$$

together with appropriate conditions on the edges of the strip.

If we suppose the displacement prescribed on the edges, we have

$$
W(x, \pm h s)= \pm p(x), \quad-\infty<x<\infty .
$$

Next introduce a harmonic function $W_{1}(x, y)$, odd in $y$, such that

$$
W_{1}(x, \pm h s)= \pm p(x)
$$

and set

$$
W=W_{1}+W_{0} .
$$

Then, using symmetry, (2.7) to (2.9) reduce to finding an harmonic function $W_{0}(x, y)$ in $(-\infty, \infty) \times(0, h s)$ such that

$$
\begin{gathered}
W_{0}(x, h s)=0, \quad-\infty<x<\infty, \\
\frac{\partial W_{0}}{\partial y}(x, 0)=-q(x), \quad|x|<a, \\
W_{0}(x, 0)=0, \quad|x|>a,
\end{gathered}
$$

where $q(x)=(\partial W / \partial y)(x, 0)$.

The conformal transformation

$$
\omega=\xi+i \eta=\tanh (c z), \quad z=x+i y,
$$

with $c=\pi / 2 h s$, transforms the given problem into one for the upper halfplane, $\eta>0$, such that

$$
\begin{gathered}
W_{0}(\xi, 0)=0, \quad|\xi|>\alpha, \\
\frac{\partial W_{0}}{\partial \eta}(\xi, 0)=\frac{-\hat{q}(\xi)}{c\left(1-\xi^{2}\right)}, \quad|\xi|<\alpha,
\end{gathered}
$$

with $\alpha=\tanh (c a)$. 
The appropriate solution, for $q$ even, is provided by the function of a complex variable [Gakhov [5]],

$$
\Omega(\omega)=\chi+i W_{0}=\frac{\sqrt{\omega^{2}-\alpha^{2}}}{\pi i} \int_{-\alpha}^{\alpha} \frac{P(\tau) d \tau}{\sqrt{\tau^{2}-\alpha^{2}}(\tau-\omega)},
$$

with

$$
P(\tau)=-\frac{1}{c} \int_{0}^{\tau} \frac{\hat{q}(\xi) d \xi}{1-\xi^{2}} .
$$

In the above and following we adopt the convention that where branch points occur the function is taken real and positive for $\xi$ large, $\eta \rightarrow 0^{+}$.

In the case of practical interest when $p(x)$ is a constant $p_{0}$,

$$
q(x)=p_{0} / h s, \quad W_{1}(x, y)=p_{0} y / h s .
$$

By carrying out the integrations in (2.14), (2.15) making use of suitable contours we find the explicit expression

$$
\Omega(\omega)=-\frac{p_{0}}{c h s} \ln \left\{\frac{\omega+1}{\sqrt{1-\alpha^{2}}+\sqrt{\omega^{2}-\alpha^{2}}}\right\} .
$$

3. Stresses in the strip. We focus attention on the quarter strip $0 \leq x<\infty, 0 \leq y \leq h s$. The form taken by the curves below in the remaining sections of the strip are easily seen by symmetry considerations.

Since

$$
\sigma_{y z}=\mu \frac{\partial W}{\partial y}, \quad \sigma_{x z}=\mu \frac{\partial W}{\partial x}, \quad W=W_{0}+W_{1},
$$

it follows from (2.17) that

$$
\begin{aligned}
& \sigma_{y z}=\frac{\mu p_{0}}{h s} \mathscr{R}\left\{\frac{\sinh (c z)}{\left[\sinh ^{2}(c z)-\sinh ^{2}(c a)\right]^{1 / 2}}\right\}, \\
& \sigma_{x z}=\frac{\mu p_{0}}{h s} \mathscr{I}\left\{\frac{\sinh (c z)}{\left[\sinh ^{2}(c z)-\sinh ^{2}(c a)\right]^{1 / 2}}\right\} .
\end{aligned}
$$

For computational purposes we introduce the new variables

$$
\begin{gathered}
\bar{x}=c x, \quad \bar{y}=c y, \quad \bar{a}=c a, \\
\sigma_{1}=h s \sigma_{y z} / \mu p_{0}, \quad \sigma_{2}=h s \sigma_{x z} / \mu p_{0},
\end{gathered}
$$

so that

$$
\sigma_{1}=\mathscr{R} F(\bar{z}), \quad 0 \leq \bar{x}<\infty, \quad \sigma_{2}=\mathscr{I} F(\bar{z}), \quad 0 \leq \bar{y} \leq \pi / 2 .
$$

To obtain representative graphs we have set $\bar{a}=1$, and plotted level curves of $\sigma_{1}, \sigma_{2}$ in the $(x, y)$ plane as shown in Figs. 1 and 2 . These have been curtailed at $\bar{x}=2$ since the behavior beyond this line is obvious. Evidently the two sets of curves are orthogonal trajectories of each other.

4. Remarks on a second problem. A second problem of interest is that in which the shear stress is given on the edges 


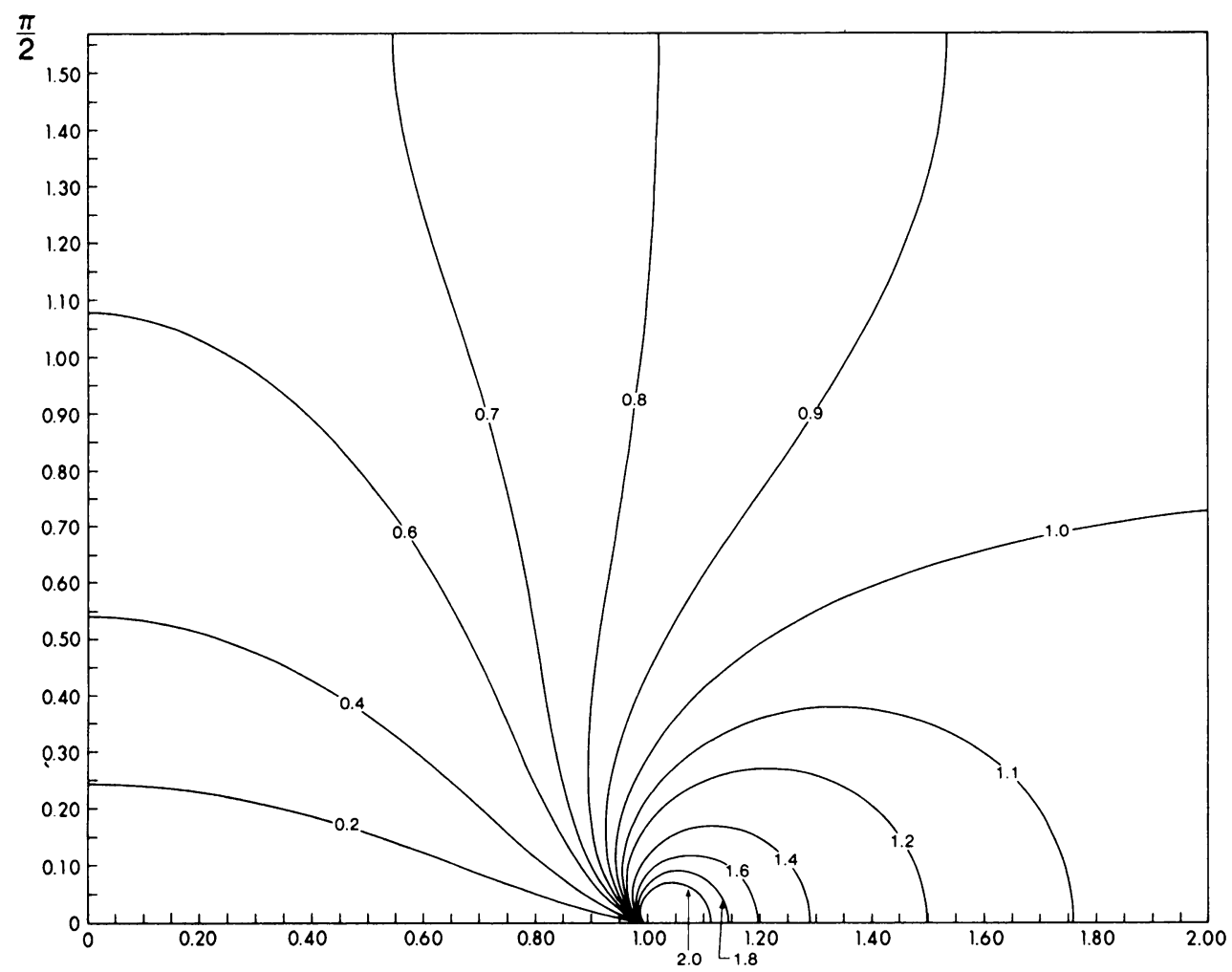

Fig. 1. Level curve of $\sigma_{1}$.

$$
\frac{\partial W}{\partial y}(x, \pm h s)=p(x) / \mu s .
$$

It is slightly more convenient to pose the problem in terms of $\zeta=\partial W / \partial y$, in which case we require a function harmonic in the strip with

$$
\begin{aligned}
\zeta(x, \pm h s) & =p(x) / \mu s, \quad-\infty<x<\infty, \\
\zeta(x, 0) & =0, \quad|x|<a, \quad \frac{\partial \zeta}{\partial y}(x, 0)=0, \quad|x|>a .
\end{aligned}
$$

If again we set $\zeta=\zeta_{1}+\zeta_{0}$, where $\zeta_{1}$ is harmonic, even in $y$ with $\left(\partial \zeta_{1} / \partial y\right)(x, 0)=0, \zeta_{1}(x$, $\pm h s)=p(x) / \mu s$, we reduce the problem to finding a harmonic function $\zeta_{0}$, with

$$
\begin{aligned}
& \zeta_{0}(x, h s)=0, \quad-\infty<x<\infty, \\
& \zeta_{0}(x, 0)=-q(x), \quad|x|<a, \quad \frac{\partial \zeta_{0}}{\partial y}(x, 0)=0, \quad|x|>a,
\end{aligned}
$$

where $q(x)=\zeta_{1}(x, 0)$, and symmetry has again been used.

Proceeding as before, we obtain

$$
\Omega(\omega)=\lambda+i \zeta_{0}=-\frac{1}{\pi}\left(\frac{\omega^{2}-1}{\omega^{2}-\alpha^{2}}\right)^{1 / 2} \int_{-\alpha}^{\alpha} \hat{q}(\tau)\left(\frac{\tau^{2}-\alpha^{2}}{\tau^{2}-1}\right)^{1 / 2} \frac{d \tau}{\tau-\omega},
$$

where $\omega=\xi+i \eta=\tanh (c z), z=x+i y$, and $\alpha=\tanh (c a)$. If again $p(x)=p_{0}$, a constant, $q(x)=p_{0} / \mu s$ then 


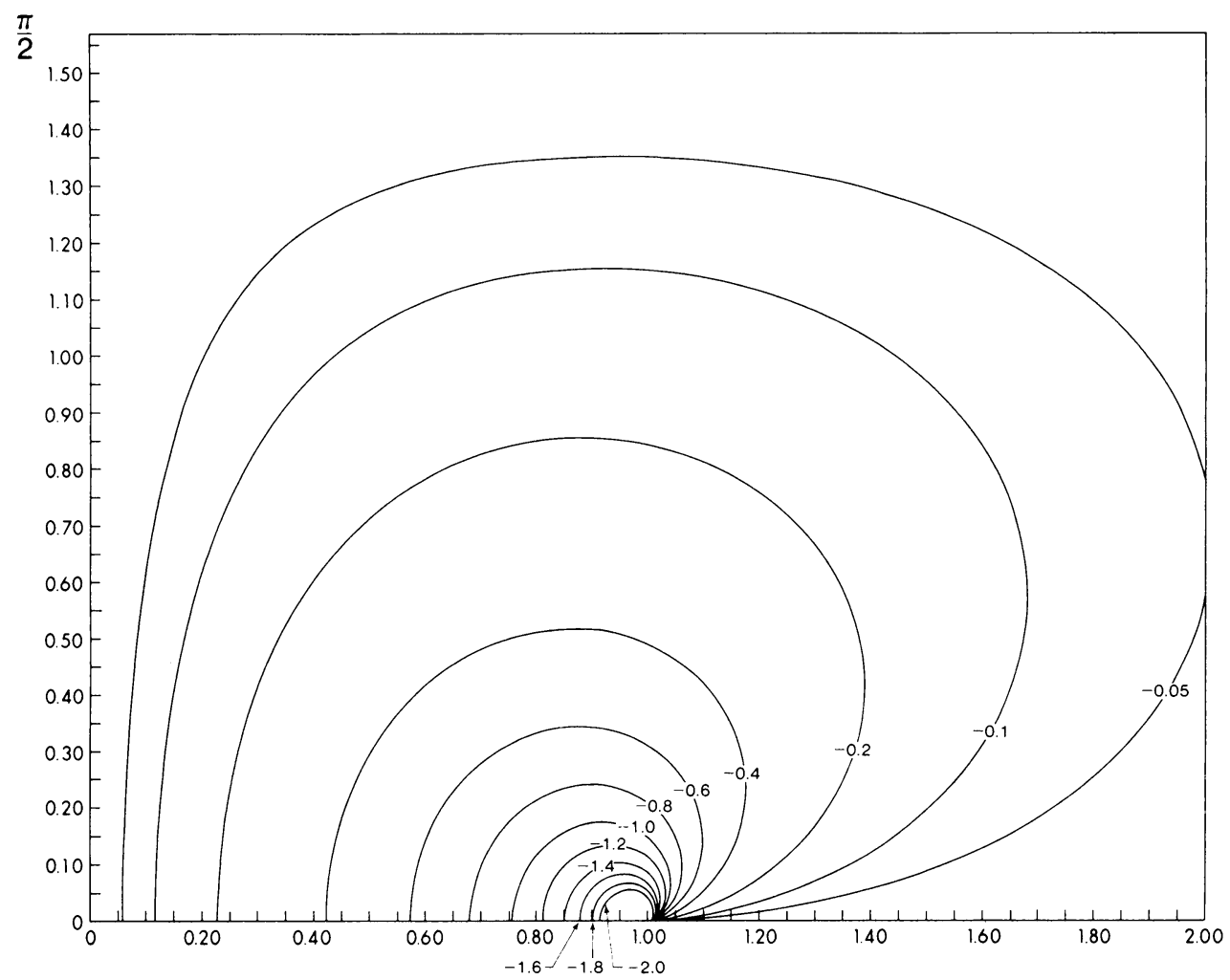

Fig. 2. Level curves of $\sigma_{2}$.

$$
\sigma_{y z}=\frac{p_{0}}{s}\left\{1-\frac{1}{\pi} \mathscr{I}\left[\left(\frac{\omega^{2}-1}{\omega^{2}-\alpha^{2}}\right)^{1 / 2} \int_{-\alpha}^{\alpha}\left(\frac{\tau^{2}-\alpha^{2}}{\tau^{2}-1}\right)^{1 / 2} \frac{d \tau}{\tau-\omega}\right]\right\} .
$$

It is possible to formally write (4.5) as

$$
\sigma_{y z}=\frac{p_{0}}{s}\left\{1-\frac{2}{\pi} \mathscr{I}\left[\frac{\left(\omega^{2}-\alpha^{2}\right)^{1 / 2}\left(\omega^{2}-1\right)^{1 / 2}}{\omega} \Pi\left(\frac{\pi}{2},-\frac{\alpha^{2}}{\omega^{2}}, \alpha\right)-\omega\left(\frac{\omega^{2}-\alpha^{2}}{\omega^{2}-1}\right)^{1 / 2} K(\alpha)\right]\right\},
$$

where $K, \Pi$ denote complete elliptic integrals of the first and third kinds, respectively. There does not appear to be any further reduction to elementary functions, and care must be taken to interpret the integrals as principal value integrals when $\omega$ is real, $|\omega|<\alpha$. The corresponding level curves would then be more difficult to obtain numerically.

\section{REFERENCES}

[1] R. J. Tait and T. B. Moodie, Complex variable methods and closed-form solutions to dynamic crack and punch problems in the classical theory of elasticity, Int. J. Eng. Sc. 19, 221-229 (1981)

[2] B. M. Singh, T. B. Moodie, and J. Haddow, Closed-form solutions for finite-length crack moving in a strip under anti-plane shear stress, Acta Mech. 38, 99-109 (1981)

[3] G. C. Sih and E. P. Chen, Mechanics of fracture, in Elastodynamic crack problems, ed. G. C. Sih, Noordhoff, International Publishing (1977), Vol. 4, Chap. 2

[4] I. N. Sneddon and M. Lowengrub, Crack problems in the classical theory of elasticity, SIAM Series in Applied Mathematics (1969)

[5] F. D. Gakhov, Boundary value problems, Pergammon Press (1966) 